\title{
Successful Treatment of Recurrent Candidemia due to Candidal Thrombophlebitis Associated with a Central Venous Catheter Using a Combination of Fosfluconazole and Micafungin
}

\author{
Hideharu Hagiya $^{1}$ and Hiroki Kajioka ${ }^{2}$
}

\begin{abstract}
We herein report the case of an 85-year-old woman presenting with right internal jugular vein candidal thrombophlebitis associated with central venous catheters (CTCVC). The infecting agent was Candida albicans, which caused recurrent candidemia five times in total. Micafungin (MCFG) alone was ineffective; however, the combination of MCFG with fosfluconazole (F-FLCZ) successfully treated the patient without a need for any anticoagulant or surgical therapies. To the extent of our knowledge, this is the first report of CTCVC being successfully treated with a combination of F-FLCZ and MCFG. These new antifungal agents have better efficacy, tolerability and bioavailability; therefore, they can be useful alternatives to classical combination therapies such as amphotericin-B and 5-fluorocytosine.
\end{abstract}

Key words: candidemia, thrombophlebitis, fluconazole, micafungin

(Intern Med 52: 2139-2143, 2013)

(DOI: 10.2169/internalmedicine.52.0584)

\section{Introduction}

A central venous catheter (CVC) provides the vascular access that is necessary for intravenous therapy, blood sampling and blood pressure monitoring. However, the use of a CVC can be associated with various complications; chiefly, the relatively common formation of a venous thrombus. Alone, this condition causes very few clinical symptoms (1), but the presence of a venous thrombus increases the risk of catheter-associated septic thrombophlebitis (2). Generally, proper surgical intervention and antimicrobial therapy are considered to be essential for the treatment of catheterassociated septic thrombophlebitis. However, candidal thrombophlebitis associated with central venous catheters (CTCVC) is a rare condition, and the most appropriate therapeutic strategy has yet to be described.

At present, the therapeutic antifungal classes that are available for the treatment of candidiasis include the polyenes, azoles and echinocandins. For candidemia, the guidelines recommend echinocandin or fluconazole (FLCZ) $(3,4)$ as initial therapy, and liposomal amphotericin B as an alternative. The effectiveness of the combination therapy of these antifungal agents has not been described, and the need for surgical resection of the infectious thrombus remains controversial. We herein report a case of CTCVC caused by Candida albicans that induced five incidences of recurrent candidemia in total, and was successfully treated with an antifungal combination therapy of fosfluconazole (F-FLCZ), a prodrug of fluconazole that is widely used as an antifungal agent in Japan, and micafungin (MCFG) without the need for surgical intervention.

\section{Case Report}

An 85-year-old woman with a past medical history of right diaphragmatic hernia was admitted to our hospital, presenting with a stomachache. A physical examination revealed tenderness in the right upper quadrant of the stomach, and abdominal ultrasound and computed tomography (CT) detected choledocholithiasis and gallbladder stones. Endoscopic retrograde cholangiopancreatography and subse-

${ }^{1}$ Emergency Unit and Critical Care Center, Tsuyama Central Hospital, Japan and ${ }^{2}$ Department of Surgery, Tsuyama Central Hospital, Japan Received for publication March 16, 2013; Accepted for publication May 1, 2013 Correspondence to Dr. Hideharu Hagiya, e_dai_for_all@hotmail.com 


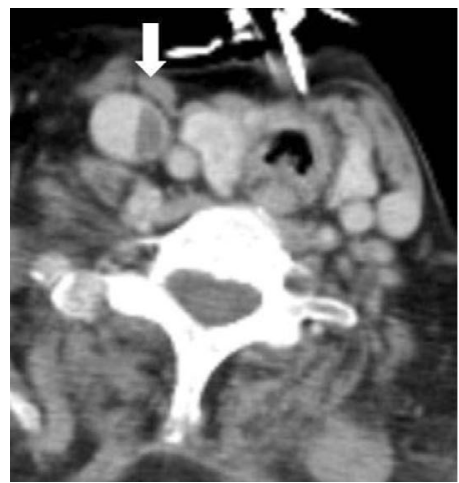

(a)

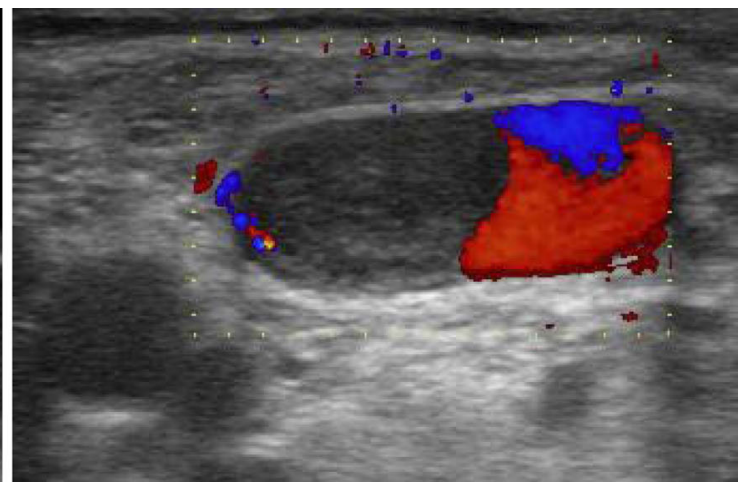

(b)

Figure 1. Thrombus inside the right jugular vein on postoperation day 38. (a) Contrast-enhanced computed tomography. (b) Echogram of the thrombus; the deficit of a colored Doppler echo suggests the existence of a thrombus.

Table. Antifungal Susceptibility Testing of Candida albicans

\begin{tabular}{|c|c|c|}
\hline \multicolumn{3}{|c|}{$\mathrm{MIC}(\mu \mathrm{g} / \mathrm{mL})$} \\
\hline Amphotericin-B & 0.5 & \\
\hline Fluconazole & 0.5 & $\mathrm{~S}$ \\
\hline Itraconazole & 0.125 & $\mathrm{~S}$ \\
\hline Voriconazole & 0.015 & $\mathrm{~S}$ \\
\hline Micafungin & 0.25 & S \\
\hline
\end{tabular}

quent endoscopic sphincterotomy were performed. During the procedure, duodenal perforation occurred and an emergent duodenojejunostomy and cholecystectomy were thus performed. As she was placed under a complete fast as a result of these procedures, a CVC was inserted into the right jugular vein on the day of surgery, and total parenteral nutrition was started.

On post-operation day (POD) 7, a localized exudate leakage into the intraperitoneal cavity was detected around the tube that was inserted into the common bile duct. She was conservatively treated with ampicillin/sulbactam for two weeks and with ciprofloxacin during the third week.

On POD 24, she suddenly developed a high fever and her right upper extremity was found to be more edematous than her left. Her white blood cell (WBC) count was elevated to $15,800 / \mathrm{mm}^{3}$, and her C-reactive protein (CRP) level was $11.8 \mathrm{mg} / \mathrm{dL}$. We suspected a central line-associated blood stream infection; thus, the CVC was immediately removed and two sets of blood culture were obtained. The next day, the blood cultures revealed the presence of a yeast-like fungus, so we administered MCFG at $100 \mathrm{mg}$ per day intravenously as an empirical therapy. C. albicans, which is sensitive to MCFG, was later isolated (Table).

A follow-up blood culture obtained on POD 30 (6 days after the first episode of candidemia) was again positive for C. albicans. The organism was also detected in the urine and in the bile that was obtained from the drainage tube inserted into the common bile duct. However, the patient did not present any symptoms or abnormal findings relating to a urinary tract infection, cholangitis or peritonitis. A transthoracic echocardiogram yielded negative findings for infective endocarditis. MCFG was continued; however, a third episode of candidemia was detected from the blood culture on POD 32 (8 days after the first episode of candidemia) and a fourth episode was detected on POD 34 (10 days after the first episode of candidemia). We had not reinserted the CVC that had been completely removed on POD 24. The results of a laboratory examination revealed a high inflammatory state (WBC: $15,800 / \mathrm{mm}^{3}$, CRP: $7.0 \mathrm{mg} / \mathrm{dL}$ ) on POD 33. We considered the possibility of cholangitis due to $C$. albicans, and combined itraconazole (ITCZ) with MCFG on POD 34. ITCZ was administered intravenously (200 mg every $12 \mathrm{~h}$ ) for one day, and was then administered orally (200 mg every $24 \mathrm{~h}$ ) because her renal function was deteriorating (creatinine: $1.04 \mathrm{mg} / \mathrm{dL}$, estimated glomerular filtration rate: $38.2 \mathrm{~mL} \cdot \mathrm{min}^{-1} \cdot 1.73 \mathrm{~m}^{-2}$ ).

Although we had deferred contrast-enhanced CT (CECT) because of her insufficient renal function, we decided to perform the scan on POD 38 to determine the focus of the recurrent candidemia. The CECT revealed thrombus formation inside the right jugular vein where the CVC had been inserted, extending from the right jugular vein to the superior vena cava, but not to the right atrium (Fig. 1 (a)). A color Doppler echogram also showed the thrombus inside the vein (Fig. 1 (b)). However, there were no inflammatory signs, such as tenderness, swelling, redness, wall thickening of the vein or fluid retention around the vein. Thrombus formations at CVC insertion sites are often seen in clinical situations and this seemingly non-inflammatory thrombus was considered as such. However, regardless of the MCFG and ITCZ combination therapy, we detected C. albicans in the blood culture for the fifth time on POD 41 (17 days after the first candidemia episode). At this juncture, we suspected CTCVC to be the cause of the recurrent candidemia.

We performed an echogram-guided needle aspiration on the thrombus. Purulent whitish material was aspirated from the inside of the thrombus, and Gram staining disclosed a 


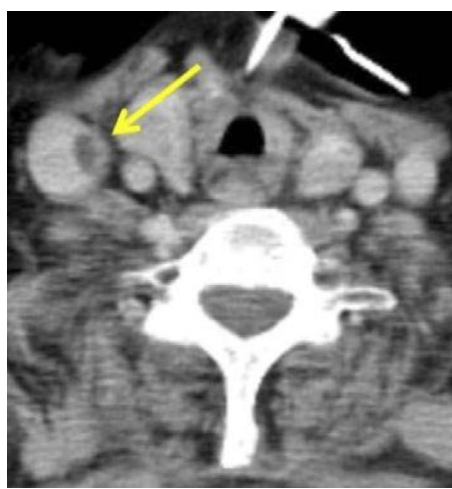

(a)

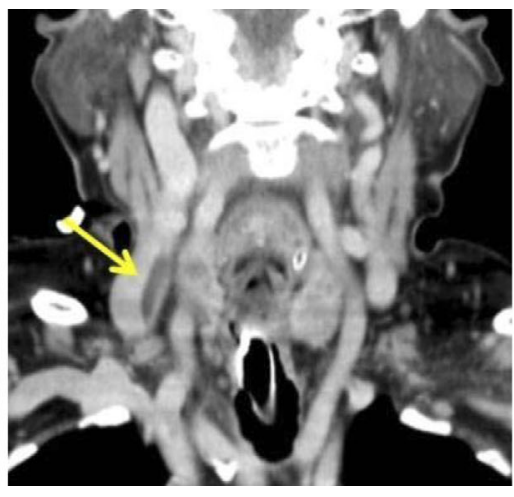

(b)

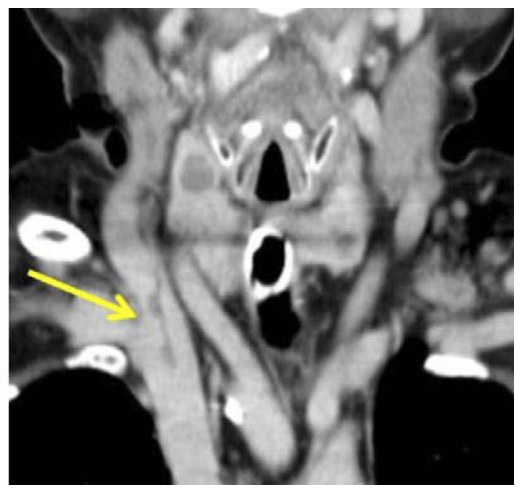

(c)

Figure 2. Contrast enhanced computed tomography (CECT) of the cervical jugular vein on postoperation day 53. Arrow indicates the thrombus. (a) Axial section. (b, c) Coronal section. CECT showing the reduction in the size of the thrombus; however, it still extended to the right brachiocephalic vein (c).

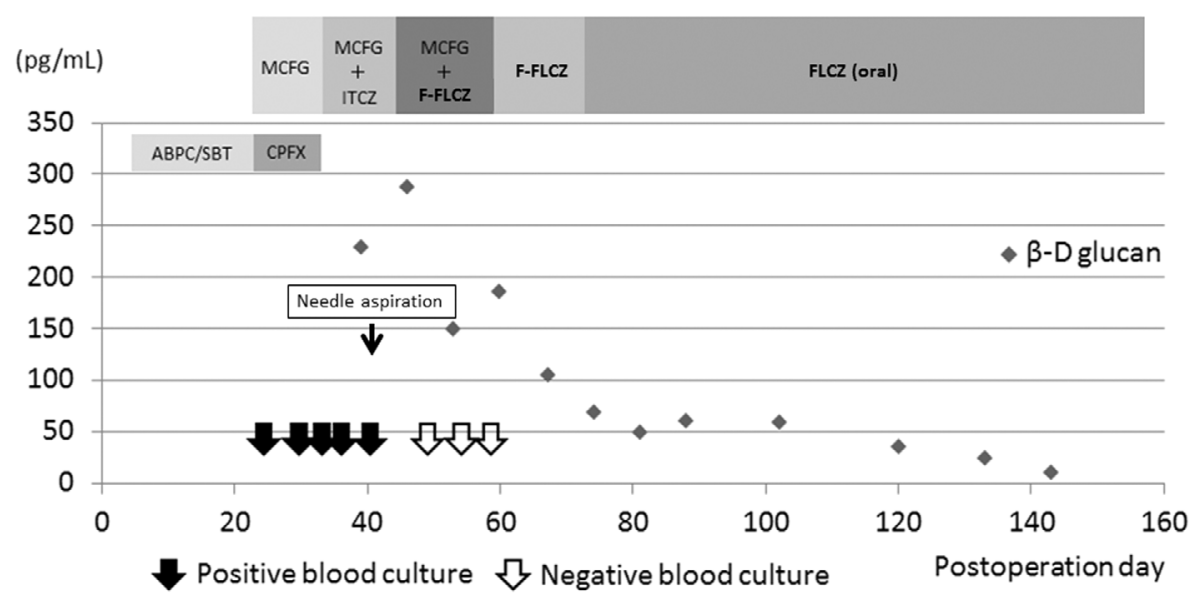

Figure 3. The clinical course of the patient. ABPC/SBT: ampicillin/sulbactam, CPFX: ciprofloxacin, MCFG: micafungin, ITCZ: itraconazole, F-FLCZ: fosfluconazole, FLCZ: fluconazole

yeast-like fungus with inflammatory cells. Subsequently, $C$. albicans was cultured from this pus and the diagnosis of CTCVC caused by $C$. albicans was confirmed.

ITCZ therapy was replaced with intravenous F-FLCZ on POD 42. F-FLCZ was administered at 3 doses of $400 \mathrm{mg}$ every 12 hours, then at 1 dose of $200 \mathrm{mg}$ every 24 hours based on her renal function. We considered surgical removal of the thrombus. However, surgical intervention was postponed because laboratory testing appeared to indicate an improvement in the symptoms; the follow-up blood culture was negative, and CECT demonstrated that the thrombus had shrunk.

Septic emboli were not seen in the lungs. The ocular fundus was examined several times throughout the treatment course and was found negative for endophthalmitis. Anticoagulant therapy was not induced at all. The $\beta$-D-glucan level increased to a maximum of $288 \mathrm{pg} / \mathrm{mL}$ on POD 46 but gradually decreased after F-FLCZ was added to the MCFG therapy.

The combination therapy of F-FLCZ and MCFG was con- tinued for two weeks. The patient displayed a good clinical course; therefore, antifungal therapy was stepped down to $400 \mathrm{mg}$ of oral fluconazole alone per day. A follow-up echogram and CECT revealed a reduction in the size of the thrombus (Fig. 2), and we confirmed by CECT that it had disappeared by POD 145 . Her $\beta$-D-glucan level also gradually returned to the normal range. In total, we administered 18 weeks of antifungal therapy without any anticoagulant therapy or surgical intervention, and the patient was discharged without any sequelae. No recurrence has since been observed.

The clinical course is summarized in Fig. 3.

\section{Discussion}

Patients with jugular vein suppurative thrombophlebitis often complain of cervical pain and present with characteristic tenderness and swelling over the mandibular angle or along the sternocleidomastoid muscle (5). In CTCVC, however, these distinctive clinical symptoms are rarely ob- 
served $(6,7)$. Instead, a persistent fever during or after the use of broad-spectrum antibiotics, recurrent detections of candidemia despite the use of antifungal agents and edema of the affected limb are considered to be the prominent clinical features of CTCVC (8). Epidemiologically, CTCVC is very rare, so the clinical suspicion tends to be delayed, which likely contributes to the mortality rate of approximately $20 \%$ (8-10). Therefore, high risk patients should be screened for CTCVC in the early phase of the clinical course. An echo-Doppler examination of all previously catheterized central veins or systemic CECT are highly recommended for long-term hospitalized patients with a fever of unknown origin, in order to detect a possible thrombus that is not clinically apparent $(8,11)$. Since the clinical picture of the patient in the present case displayed the characteristics for CTCVC (critically ill surgical patient, intraabdominal infection and long-duration antibiotics treatment), the definitive diagnosis should have been made earlier.

Although radical excision of the affected vein plays an important role in the treatment of peripheral candidal thrombophlebitis (12-14), CTCVC excision is controversial. Some reports indicate that surgical thrombectomy should be considered as a therapeutic option $(15,16)$, but others do not (8). Medical therapy alone may be effective in some cases and surgery is not always possible because of the inaccessibility of the central veins (17-20). However, Caccese et al. reported the mortality of those receiving medical therapy to only be $13 \%$, while no deaths were reported among those treated with combined medical and surgical therapy (10); thus, the authors concluded that the decision for combined surgical intervention should be reassessed. Considering the inaccessibility of the thrombus, the risk of operation and the good clinical course with the antifungal therapy alone, we therefore decided against surgery in this case.

Systemic anticoagulant or thrombolytic therapy has been used as adjunctive therapy, but there are insufficient data to recommend their use routinely (3). Heparin has been used in cases of bacterial thrombophlebitis (20), but appears to have no additional beneficial effect (18). Some medical professionals favor anticoagulation only if there is evidence for an extension of the thrombus (21). In this case, a tube was inserted into the common bile duct and the initiation of anticoagulant therapy was abandoned. Since the thrombus shrank with antifungal therapy only, the antifungal treatment was continued without anticoagulation even after the tube was removed.

Although resistance has been reported previously $(22,23)$, C. albicans, the pathogen isolated most frequently from CTCVC $(9,10)$, is generally susceptible to MCFG. In the present case, we administered MCFG first as an empirical therapy. The fungicidal activity of echinocandins against most Candida spp. in vitro is high, and it is considered to be effective even in the presence of biofilms $(24,25)$. Therefore, MCFG was expected to be the better choice for CTCVC. However, as treatment failure with caspofungin was reported (26), we selected combination therapy instead of MCFG monotherapy following the diagnosis of CTCVC.

During the course of treatment, we suspected candidal cholangitis as the cause of the recurrent candidemia. Although the definitive data are poor, ITCZ is considered to penetrate the bile relatively well compared to the other azoles, thus we combined ITCZ with MCFG. However, the candidemia persisted and this combination failed as well. We considered the route of administration to be the reason for the treatment failure. ITCZ was administered orally with a consideration to the additional renal impairment caused by the accumulation of the cyclodextrin that is mixed with the intravenous ITCZ. It was believed that the drug was not well absorbed from the intestine since the patient had recently undergone abdominal operation and the bile duct was continuously drained.

After the diagnosis of CTCVC was made, F-FLCZ was combined with the MCFG therapy. Although liposomal amphotericin B would have been preferred per the current guidelines (3), it was not chosen because of its high cost and the potential for various side effects. FLCZ interferes with the fungal cytochrome P450 activity (lanosterol 14- $\alpha$ demethylase) and decreases ergosterol synthesis, while MCFG is a concentration-dependent inhibitor of 1,3-beta-Dglucan synthase. Therefore, their antifungal mechanisms are entirely different and their combination was considered to be effective. Candidemia was successfully controlled by this combination therapy and the antifungal agent was eventually changed to oral FLCZ alone. The reason MCFG alone was ineffective is not yet clear. Although the pathogenic organism was sensitive to MCFG, its minimum inhibitory concentration (MIC) was relatively higher $(0.25 \mu \mathrm{g} / \mathrm{mL})$ and, therefore, high dose therapy (e.g., $300 \mathrm{mg}$ of MCFG) would have been needed.

CTCVC is considered a superficial Candida infection of the involved thrombus, therefore, antifungal therapy alone is considered to be effective (27). However, in this case, a hypoechoic area was detected inside the thrombus and purulent material containing inflammatory cells and C. albicans was aspirated from the inside. Therefore, we consider CTCVC an infection of not only the surface of the thrombus, but also of its interior. It was possible that the newly formed drug route to the thrombus made by the needle aspiration that was performed on POD 41, and not the combination therapy itself, may have yielded a better therapeutic outcome.

The effectiveness of combination therapy against CTCVC has been reported previously; however, these cases involved combinations of amphotericin-B and 5-fluorocytosine (8). To the extent of our knowledge, this is the first report of CTCVC being successfully treated with combination therapy including F-FLCZ and MCFG. The efficacy, tolerability and bioavailability of these new antifungal agents are better; therefore, they can be useful alternatives to the previously reported treatments (26).

In summary, CTCVC should be considered when candidemia persists despite ongoing antifungal therapy and the re- 
moval of the CVC. The combination therapy of F-FLCZ and MCFG successfully controlled the recurrent candidemia caused by CTCVC without requiring surgical thrombectomy or anticoagulant therapy. The efficacy of the combination therapy of these new antifungal agents against candidemia should be verified through further study.

The authors state that they have no Conflict of Interest (COI).

\section{References}

1. Chastre J, Cornud F, Bouchama A, Viau F, Benacerraf R, Gibert C. Thrombosis as a complication of pulmonary-artery catheterization via the internal jugular vein. $\mathrm{N}$ Engl J Med 306: 278-281, 1982.

2. Timsit JF, Farkas JC, Boyer JM, et al. Central vein catheterrelated thrombosis in intensive care patients: incidence, risks factors, and relationship with catheter-related sepsis. Chest 114: 207 213, 1998.

3. Pappas PG, Kauffman CA, Andes D, et al; Infectious Diseases Society of America. Clinical practice guidelines for the management of candidiasis: 2009 update by the Infectious Diseases Society of America. Clin Infect Dis 48: 503-535, 2009.

4. Ullmann AJ, Cornely OA, Donnelly JP, et al. ESCMID guideline for the diagnosis and management of Candida diseases 2012: developing European guidelines in clinical microbiology and infectious diseases. Clin Microbiol Infect 18 (Suppl 7): 1-8, 2012.

5. Chirinos JA, Lichtstein DM, Garcia J, Tamariz LJ. The evolution of Lemierre syndrome: report of 2 cases and review of the literature. Medicine (Baltimore) 81: 458-465, 2002.

6. Moore CL, Osaki-Kiyan P, Haque NZ, Perri MB, Donabedian S, Zervos MJ. Daptomycin versus vancomycin for bloodstream infections due to methicillin-resistant Staphylococcus aureus with a high vancomycin minimum inhibitory concentration: a casecontrol study. Clin Infect Dis 54: 51-58, 2012.

7. Rubenstein M, Creger WP. Successful streptokinase therapy for catheter-induced subclavian vein thrombosis. Arch Intern Med 140: 1370-1371, 1980.

8. Benoit D, Decruyenaere J, Vandewoude K, et al. Management of candidal thrombophlebitis of the central veins: case report and review. Clin Infect Dis 26: 393-397, 1998.

9. Meda MS, Lopez AJ, Guyot A. Candida inferior vena cava filter infection and septic thrombophlebitis. Br J Radiol 80: e48-e49, 2007.

10. Caccese R, Carfagna P, Pistilli N, Massetti AP, Falcone M, Venditti M. Candidal thrombophlebitis of central veins: case report and review. Med Mycol 50: 299-304, 2012.

11. Tzortzis S, Apostolakis S, Xenakis K, Spiropoulos G, Lazaridis K. Catheter-related septic thrombophlebitis of the superior vena cava involving the atrial septum: a case report. Cases J 1: 272, 2008.

12. Hong SK, Nam SH, Kim HC. Fatal peripheral candidal suppura- tive thrombophlebitis in a postoperative patient. J Korean Med Sci 23: 1094-1096, 2008.

13. Torres-Rojas JR, Stratton CW, Sanders CV, et al. Candidal suppurative peripheral thrombophlebitis. Ann Intern Med 96: 431-435, 1982.

14. Hauser CJ, Bosco P, Davenport M, Ehrensaft D, Latif M, McNamara BT. Surgical management of fungal peripheral thrombophlebitis. Surgery 105: 510-514, 1989.

15. Kelly RF, Yellin AE, Weaver FA. Candida thrombosis of the innominate vein with septic pulmonary emboli. Ann Vasc Surg 7: 343-346, 1993.

16. Garcia E, Granier I, Geissler A, Boespflug MD, Magnan PE, Durand-Gasselin J. Surgical management of Candida suppurative thrombophlebitis of superior vena cava after central venous catheterization. Intensive Care Med 23: 1002-1004, 1997.

17. Jarrett F, Maki DG, Chan CK. Management of septic thrombosis of the inferior vena cava caused by Candida. Arch Surg 113: 637639, 1978.

18. Strinden WD, Helgerson RB, Maki DG. Candida septic thrombosis of the great central veins associated with central catheters. Ann Surg 202: 653-658, 1985.

19. Paige C, Pinson CW, Antonovic R, Strausbaugh LJ. Catheterrelated thrombophlebitis of the superior vena cava caused by Candida glabrata. West J Med 147: 333-335, 1987.

20. Verghese A, Widrich WC, Arbeit RD. Central venous thrombophlebitis: the role of medical therapy. Medicine 64: 394-400, 1985.

21. Golpe R, Marín B, Alonso M. Lemierre's syndrome (necrobacillosis). Postgrad Med J 75: 141, 1999.

22. Hernandez S, López-Ribot JL, Najvar LK, McCarthy DI, Bocanegra R, Graybill JR. Caspofungin resistance in Candida albicans: correlating clinical outcome with laboratory susceptibility testing of three isogenic isolates serially obtained from a patient with progressive Candida esophagitis. Antimicrob Agents Chemother 48: 1382-1383, 2004.

23. Kofteridis DP, Lewis RE, Kontoyiannis DP. Caspofungin-nonsusceptible Candida isolates in cancer patients. J Antimicrob Chemother 65: 293-295, 2010.

24. Morace G, Borghi E, Iatta R, Montagna MT. Anidulafungin, a new echinocandin: in vitro activity. Drugs 69 (Suppl 1): 91-94, 2009.

25. Lye DC, Hughes A, O'Brien D, Athan E. Candida glabrata prosthetic valve endocarditis treated successfully with fluconazole plus caspofungin without surgery: a case report and literature review. Eur J Clin Microbiol Infect Dis 24: 753-755, 2005.

26. Pan SC, Hsieh SM, Chang SC, Lee HT, Chen YC. Septic Candida krusei thrombophlebitis of inferior vena cava with persistent fungemia successfully treated by new antifungal agents. Med Mycol 43: 731-734, 2005.

27. Hoffman MJ, Greenfield LJ. Central venous septic thrombosis managed by superior vena cava Greenfield filter and venous thrombectomy: a case report. J Vasc Surg 4: 606-611, 1986.

(C) 2013 The Japanese Society of Internal Medicine http://www.naika.or.jp/imonline/index.html 